\title{
e-Phaïstos
}

e-Phaïstos

Revue d'histoire des techniques / Journal of the history

of technology

VII-2 | 2019

La sauvegarde du patrimoine scientifique

\section{Articuler l'histoire des sciences et l'histoire des techniques : une approche heuristique.}

La question de la matière entre sciences et techniques (XIX ${ }^{\mathrm{e}}-\mathrm{XX}^{\mathrm{e}}$ siècles)

Articulating the History of Science and the History of Technology: a Heuristic

Approach: The Material Studies between Science and Technology

\section{Virginie Fonteneau}

\section{(2) OpenEdition \\ Journals}

\section{Édition électronique}

URL : http://journals.openedition.org/ephaistos/5092

DOI : 10.4000/ephaistos.5092

ISSN : 2552-0741

\section{Éditeur}

IHMC - Institut d'histoire moderne et contemporaine (UMR 8066)

\section{Référence électronique}

Virginie Fonteneau, «Articuler l'histoire des sciences et l'histoire des techniques : une approche

heuristique. », e-Phaïstos [En ligne], VII-2 | 2019, mis en ligne le 15 octobre 2019, consulté le 09 novembre 2019. URL : http://journals.openedition.org/ephaistos/5092

Ce document a été généré automatiquement le 9 novembre 2019.

Tous droits réservés 


\title{
Articuler l'histoire des sciences et l'histoire des techniques : une approche heuristique.
}

La question de la matière entre sciences et techniques (XIX ${ }^{\mathrm{e}}-\mathrm{XX}^{\mathrm{e}}$ siècles)

Articulating the History of Science and the History of Technology: a Heuristic Approach: The Material Studies between Science and Technology

\section{Virginie Fonteneau}

\author{
Habilitation à diriger des recherches : références bibliographiques \\ Virginie FONTENEAU, Articuler l'histoire des sciences et l'histoire des techniques : \\ une approche heuristique. La question de la matière entre sciences et techniques \\ (XIX ${ }^{\mathrm{e}}-\mathrm{XX}^{\mathrm{e}}$ siècles), Habilitation à diriger des recherches, Université Paris-Sud, \\ soutenue le 12 octobre 2017. \\ Jury : \\ Jose-Ramon BERTOMEU-SANCHEZ, Université de Valence \\ Alain FUCHS, CNRS \\ Anne-Françoise GARÇON, Université Paris 1 Panthéon-Sorbonne \\ Hélène GISPERT, Université Paris-Sud \\ André GRELON, EHESS \\ Pierre LAMARD, Université de Technologie de Belfort-Montbéliard \\ Laurence LESTEL, CNRS
}

1 Madame la Présidente, mesdames et messieurs les membres du jury,

2 A l'occasion de cette soutenance, j'ai eu envie - mais peut-être était-ce plutôt une nécessité - de partager avec vous une de mes dernières lectures. L'ouvrage n'est pas récent (édition première en 2009), il s'agit du livre de Matthew Crawford, Éloge du carburateur, essai sur le sens et la valeur du travail ${ }^{1}$, que certains d'entre vous connaissent 
puisqu'il a connu un large écho. Crawford y porte une réflexion sur le travail et notamment, pour mon propos, sur les métiers manuels et les métiers intellectuels.

Deux raisons particulières m'incitent à vous parler de cette lecture, la première est que celle-ci m'a éclairée sur les liens entre mon histoire, ma personnalité et mon métier, et la deuxième est qu'elle est source de réflexion pour certains de mes thèmes de recherches. Elle m'a aussi ramenée à un ouvrage synthétique d'Antoine Prost, 12 leçons sur l'histoire ${ }^{2}$ que j'avais lu au tout début de mes études d'histoire des sciences et des techniques, il y a longtemps, et que j'ai repris pour préparer cette soutenance. Je commence par l'apport personnel parce que c'est par ce cheminement réflexif que je suis arrivée ou revenue à mes objets de recherche, ce qu'A. Prost désigne comme le poids de la personnalité dans le métier d'historien: "Comme toute chance, la connaissance intime par engagement personnel est aussi un risque. Elle permet à l'historien d'aller plus vite et plus loin dans la compréhension de son sujet, mais elle peut aussi étouffer sa lucidité sous le bouillonnement de ses affects $»^{3}$. Cette lecture a été une occasion de penser et panser mes affects, de réfléchir à ce qui me permet d'aller plus vite et plus loin sur mes terrains de recherche, et, point crucial, si ou en quoi cela était justifié.

5 Pour poser sa réflexion, Crawford part de son expérience. Après un diplôme en physique, puis une thèse de philosophie politique tout en gagnant parallèlement sa vie grâce à métier manuel (électricien notamment), il fait deux expériences de travail intellectuel, une comme rédacteur de résumés d'articles scientifiques, et une dans un think tank. Découragé par un sentiment d'inutilité, de perte de sens et d'insatisfaction dans un travail dit «intellectuel ", il entame une carrière comme réparateur de moto. Dans ce livre, Crawford se concentre sur le travail manuel qualifié et pas sur l'artisanat plus valorisé. Il défend l'idée d'une « richesse intrinsèque du travail manuel du point de vue cognitif, social et psychologique $»^{4}$. Il démontre qu'en exerçant un travail manuel qualifié, il y a en même temps engagement de l'intellect. Ceci implique que lorsque l'on distingue deux types de travail, manuel et intellectuel, on disjoint le manuel de l'intellectuel et l'on donne donc à penser qu'il n'y a pas engagement de l'intellect lors d'un travail manuel qualifié. Selon lui, cette séparation forte prend son origine dans le cas des États-Unis dans l'émergence du travail à la chaine (début du XX ${ }^{e}$ siècle), et dans la mise en place, en même temps d'un enseignement technique à deux niveaux: préparation à la chaîne de montage pour les classes subalternes (c'est-à-dire, conduire à l'acceptation d'un travail manuel réduit à l'application d'une consigne), activités éducatives de type récréatif et donc valorisantes pour la bourgeoisie. Il y a parallèlement valorisation du théorique et de l'intellectuel sur le manuel.

6 Crawford explique que le Taylorisme, sous couvert de transformation de l'usine moderne en champ d'expérimentation et de vouloir amener le travailleur à une démarche scientifique, impose en fait à l'ouvrier une séparation du savoir et du faire qui loin d'emmener le travailleur dans une démarche scientifique lui confisque son autonomie. Les expressions « travail manuel » et «travail intellectuel » sont devenues des catégories usuelles. A force de généraliser l'utilisation de ces expressions, et d'intérioriser cette séparation entre le manuel et l'intellectuel, on donne à penser comme le souligne Crawford que le travail manuel est toujours de l'ordre du travail à la chaine c'est-à-dire l'application d'une consigne (et donc aussi peu intéressant et aussi peu engageant au niveau de l'intellect). Il démontre que la généralisation est fausse. En s'appuyant sur des travaux venant de plusieurs disciplines, il argumente que la 
confrontation au concret, à la matérialité, mise en œuvre dans un certain nombre de métier manuel qualifié, dont réparateur de moto, conduit à apprendre, à reconnaître, à mémoriser, à traiter quantité d'informations de nature diverse, ce qui, si l'apprentissage se passe bien, rend capable de résoudre des problèmes mais aussi d'être capable de trouver quel est le problème avant de le résoudre.

7 Crawford traite également de la connaissance tacite, «A la base (de laquelle), il y a, ditil, l'idée que nous en savons plus que nous ne sommes capables de l'exprimer, et certainement plus que nous ne sommes à même de le spécifier par une formulation rigoureuse $»^{5}$. Il y a donc construction d'une approche cognitive particulière dans la confrontation avec le réel. Il cite ainsi l'étude d'un psychologue sur le processus de décision de praticiens experts. Celui-ci affirme que «les experts apprennent à percevoir des choses qui restent invisibles pour les novices, telles que les caractéristiques d'une situation typique $»^{6}$, que "l'esprit expérimenté est souvent capable d'intégrer un nombre extraordinaire de variables et de détecter une configuration (pattern) cohérente $»^{7}$ et que « c'est cette configuration qu'il appréhende, pas les variables individuelles $»^{8}$.

8 Cette lecture m'a donné des clés de compréhension sur mon milieu familial - d'où je viens-, mais aussi sur ce qui je suis en tant que chercheuse, ce qui m'amène à nouveau à Alain Prost, et à un passage intitulé : "Autoportrait de l'historien en artisan $»^{9}$ : "Quand on lit les textes d'historiens sur l'histoire, on est frappé par la récurrence du vocabulaire artisanal $\aleph^{10}$ écrit-il. Il poursuit en pointant que l'on présente aussi l'histoire comme une science :

« Il faudrait pourtant choisir en bonne logique. La menuiserie n'est pas une science, l'atelier n'est pas un laboratoire, ni l'établi une paillasse. Les sciences s'enseignent, on peut en énoncer les règles. Or celles de l'histoire n'en sont pas vraiment bien qu'elles existent, affirme-t-on cependant. La présence dans le discours sur l'histoire de termes qui renvoient à des univers intellectuels et pratiques si différents, ne laissent pas d'interroger. La métaphore artisane revient trop souvent pour n'être qu'une simple captatio benevolentiae, ou une fausse modestie. Assurément, les historiens traduisent dans le lexique de l'artisanat un aspect essentiel de leur expérience, le sentiment très fort qu'il n'y pas de règle qu'on puisse appliquer automatiquement et systématiquement, que tout est affaire de dosage, de doigté, de compréhension. Ce qui ne les empêche pas de se vouloir et d'être rigoureux, et de l'exprimer en recourant au lexique de la science. En fait, la complexité de l'histoire comme pratique renvoie à la complexité même de son objet. $»^{11}$

Il y aurait beaucoup à dire sur ce passage, entre autres sur l'image de la science. Ce que je retiens ici c'est la complexité de l'objet de l'historien comparable à la complexité de la matérialité travaillé par le menuisier. En écho à la lecture de Crawford, les lignes d'Antoine Prost me revenaient en mémoire : cette parenté (pas identification mais bien parenté) entre travail artisanal et le métier d'historien. La longue confrontation avec son terrain de recherches, la "familiarisation" avec les archives sont une phase d'imprégnation où l'historien intègre un nombre considérable de données, qui, si l'on devient expert au sens de Crawford, permettra de reconnaitre des configurations qu'on 
n'arrive pas toujours à justifier, mais qui font sens pour l'expert et que l'historien dans une démarche méthodologique de va et vient entre ses sources primaires et ses sources secondaires va retenir ou écarter. Il n'existe pas de règle qu'on puisse appliquer automatiquement et systématiquement, c'est-à-dire qu'il n'existe pas non plus de méthodologie a priori. Celle-ci doit toujours être adaptée à l'objet étudié. Ces pistes de recherches pressenties, celles écartées ou privilégiées, pour des raisons qu'on ne peut pas toujours expliciter, permettent d'aller plus vite. Je tiens à souligner l'importance des sources secondaires car il n'est pas question de s'enfermer dans son terrain. Il y a un équilibre à trouver, « tout est affaire de dosage, doigté, compréhension ${ }^{12}$.

L'analyse de Crawford était aussi instructive à propos de mes objets de recherche. Je pense que la séparation entre travail manuel et travail intellectuel affirmée et intériorisée influe sur les travaux menés en histoire des sciences, et ce, malgré les courants récents, et les appels - notamment en histoire de la chimie - à étudier les objets, les protocoles, les lieux (laboratoires, manufactures, etc.), ce qu'on appelle le « material turn $»^{13}$. Ce tournant matériel est heuristique et permet de rendre visible des pratiques, des savoirs, des savoir-faire mais aussi des acteurs, des lieux ignorés ou minorés par l'historiographie. Cela étant, je pense qu'implicitement, on projette sur le travail d'un chimiste cette distinction entre activités manuelles et travail intellectuel. L'engagement de l'intellect n'est pas suffisamment considéré dans la pratique du chimiste. Je suis convaincue que nous n'avons pas pris, en histoire de la chimie, la mesure de ce qui résulte pour un chimiste du fait de passer des heures par jour à la paillasse, de se confronter des heures durant, des jours durant à la complexité de la matière. $\mathrm{Au}$ xix ${ }^{\mathrm{e}}$ siècle, un chimiste apprend à mémoriser des odeurs, des goûts, des réactions, des savoirs faire, ce faisant il intègre une multitude d'informations de nature diverse, parmi lesquelles il faut qu'il sélectionne. Je reprends Crawford :

\footnotetext{
«Dans le monde réel, les problèmes ne se présentent pas sous cette forme prédigérée [les problèmes que l'on doit résoudre dans son cursus]; en général, vous disposez de trop d'éléments d'information, mais sans vraiment savoir lesquels sont pertinents et lesquels ne le sont pas. Identifier à quel genre de problème vous êtes confronté vous permet de savoir quelles caractéristiques de la situation vous pouvez vous permettre d'ignorer. Et même les frontières de ce qui peut passer pour une «situation » sont parfois ambigües ; ce n'est pas en appliquant des règles que vous pouvez discriminer entre le pertinent et le négligeable, mais seulement en exerçant le type de jugement qui naît de l'expérience. $»^{14}$
}

11 Il va de soi que les historiens de la chimie n'adoptent pas tous cette dichotomie entre le faire et le savoir, mais la matérialité n'est pas encore perçue comme essentielle pour l'étude de la production de savoirs. C'est une des raisons, pas la seule, pour laquelle, méthodologiquement, je pense qu'il est fructueux de se pencher sur la littérature secondaire d'histoire des techniques avant de commencer à faire des recherches sur ces questions parce que la matérialité, le rapport à l'objet et à l'outil, à la machine, à l'instrument, sont fondamentaux et il y a donc là source d'inspiration.

12 Ceci m'amène à la dernière partie de cette présentation: comment l'articulation de l'histoire des sciences et de l'histoire des techniques a été fructueuse dès mes premiers travaux de recherches, en DEA puis en thèse. 
13 Le premier exemple vient de mon travail de thèse sur la première école d'ingénieurs créée à Nantes en 1919. Les deux premiers directeurs de l'école venaient de mondes très différents: le premier, non titulaire du baccalauréat, enseignant dans le Technique, puis, sur le tard, ingénieur d'une toute nouvelle école, l'École supérieure d'aéronautique et de constructions mécaniques; le second, titulaire du baccalauréat, puis des études de physique en faculté des sciences, et ensuite une thèse sous la direction de Gabriel Lippmann, prix Nobel de physique de 1908. Pour étudier et comprendre le parcours du premier, il fallait puiser dans l'histoire des techniques et de l'enseignement technique, et, pour le second, dans l'histoire des sciences et de l'enseignement supérieur scientifique. Ce ne fut facile ni pour l'un ni pour l'autre, les deux étant absents de l'historiographie, en raison du peu de travaux existant en histoire de l'enseignement technique supérieur pour l'un et, pour l'autre, en raison du peu d'études faites sur les institutions scientifiques de provinces. Les deux approches étaient donc nécessaires. De plus, cette connaissance m'a permis de puiser de façon fructueuse dans des sources bien connues des historiens des techniques mais peu consultés en histoire des sciences ce qui m'a permis d'éclairer le parcours du second directeur, physicien. J'ai trouvé des informations précieuses dans les fonds de brevets, et des revues spécialisées techniques que je ne serais sans doute pas allée chercher sans cette double approche.

Le deuxième exemple vient à nouveau du travail de thèse, mais il dépasse le cadre de l'histoire de l'école. Lors du changement de direction, l'ancien et le futur directeur se sont opposés sur l'avenir de l'école à propos de la recherche et de l'enseignement de la mécanique. Dans le système d'enseignement de cette période en France, ces deux personnages avaient peu de probabilités de se trouver à un même niveau de responsabilités leur donnant l'occasion de débattre de la question de la mécanique d'égal à égal, ou presque. J'aurais pu analyser cette opposition uniquement par la différence des milieux, des parcours, et de l'autorité et de la légitimité qui en découlaient mais cela aurait été insuffisant. Cette mécanique s'imposait comme une évidence dans les discours d'acteurs, alors qu'elle était loin d'être une évidence pour moi. Articuler l'histoire des techniques et l'histoire des sciences m'a permis de montrer que derrière le même mot, mécanique, ces deux acteurs avaient une conception bien différente de ce que recouvrait ce champ et de comment il fallait l'enseigner. L'un s'inscrivait dans une tradition expérimentale et appliquée dans le sillage du Conservatoire des arts et métiers, et des écoles d'arts et métiers, et dans une démarche de recherches sur les matériaux avec comme références, Léon Guillet, et Henry Le Chatelier. L'autre s'inscrivait dans l'héritage de l'École polytechnique, avec une mécanique théorique et mathématisée.

J'en viens maintenant à l'histoire de la chimie, en particulier au XIX $\mathrm{X}^{\mathrm{e}}$ siècle, où la chimie est à la fois objet de science et au cœur de procédés techniques, et de la transformation et de l'émergence de secteurs industriels. L'articulation entre histoire des sciences et histoire des techniques s'imposait à moi pour saisir mon questionnement sur les liens entre la chimie "scientifique » et la chimie technique et sur les acteurs (comment et où ces acteurs apprennent, enseignent et pratiquent la chimie ?). Je vais revenir rapidement sur certaines des conclusions, des pistes et projets de recherches du mémoire original « Des acteurs et des lieux de la chimie en France au XIX ${ }^{e}$ siècle : Création, devenir et ancrage territorial des cours publics de chimie ». 
16 Le premier point porte sur le nombre important de cours de chimie qui apparaissent dans la première partie du $\mathrm{XIX}^{\mathrm{e}}$ siècle en France, pour nombre d'entre eux avant les cours promus par Dupin. Ces cours de chimie ne sont pas un essaimage de cours parisiens qui viendraient s'implanter dans les provinces. Ce résultat montre que les provinces ne sont pas, loin de là, un désert pour la production des savoirs et l'enseignement de la chimie au cours du XIXe siècle. Le rôle de l'État centralisé n'est pas aussi déterminant que l'historiographie l'a présenté jusqu'à présent pour la mise en place des enseignements de chimie. Il y a une marge de manœuvre invisible lorsque l'on étudie le cadre prescriptif donné par l'État ${ }^{15}$.

17 Ce travail met aussi en évidence le fait que bon nombre de chimistes circulent d'une ville provinciale à une autre ville provinciale, contrairement à l'idée implicite que les circulations se font prioritairement entre les villes de provinces et Paris. Ceci nécessite que l'information sur les cours, sur les postes, soit diffusée dans toute la France. Une étude spécifique reste à mener sur les motivations, les modalités de circulation de ces chimistes, ce qui permettrait de dresser une cartographie dynamique de la chimie et de ses acteurs. Les études de cas (Lyon, Lille, Rouen et Bordeaux) permettent de montrer qu'il y a des scenarii différents de mise en place et d'institutionnalisation de l'enseignement de la chimie au cours du XIX ${ }^{\mathrm{e}}$ siècle, et que la situation de la fin du siècle ne prend sens qu'avec les éléments de compréhension du début de siècle.

La préparation de ce mémoire original a renforcé ma conviction de la richesse que représenterait l'étude des chimistes peu connus du XIXe siècle parce que loin, pour la plupart, des élites académiques et parce que les traces qu'ils laissent sont disparates, dispersées et tenues. Mener un travail biographique ou prosopographique représente un défi tant qu'il faut relever car il permettrait de dessiner un nouveau (ou de nouveaux) profil(s) des chimistes du XIX ${ }^{e}$ siècle, tour à tour ou en même temps, enseignant, inventeur, entrepreneur, auteur, médecin ou pharmacien, très souvent investi dans une ou plusieurs sociétés savantes. Par l'étude de ces carrières, il y aurait une des clés de la compréhension du milieu de la chimie au cours du XIX ${ }^{\mathrm{e}}$ siècle.

19 La rédaction de ce manuscrit a été aussi source de plusieurs frustrations. La première est d'avoir finalement peu travaillé sur l'espace du laboratoire, central dans la vie du chimiste, que j'aimerais traiter sous deux angles peu traités: le budget, car un laboratoire de chimie ne fonctionne plus s'il n'y a pas du charbon pour les fours, si la verrerie n'est pas remplacée, sans matières premières, sans personnel, etc. ; l'étude de l'ensemble des acteurs présents au laboratoire de chimie donc y compris les préparateurs, qui sont loin de se destiner tous à une carrière d'enseignement ou de recherche quand on y regarde d'un peu plus près. Nombre d'entre eux se lancent dans une activité industrielle, souvent petite et, ils sont souvent absents des études sur les entreprises de la chimie. La seconde frustration est celle du temps qui passe et du nombre de questions qu'il faudrait traiter, dont beaucoup nécessiteraient un travail collectif alors que la communauté des historiens de la chimie est certes dynamique mais réduite, ce qui rend difficile la conduite de programmes collectifs majeurs.

Je prendrais deux exemples dont les enjeux vont au-delà du seul domaine de la chimie. Tout d'abord, dans les questions d'enseignement et de formation, il serait important d'étudier la transition d'un système où, la certification n'est pas nécessaire aux chimistes au XIX ${ }^{\mathrm{e}}$ siècle à un système où la certification devient indispensable. Au XIX ${ }^{\mathrm{e}}$ siècle, les lieux de formations à la chimie sans certification sont nombreux : Muséum d'histoire naturelle, Conservatoire des arts et métiers, laboratoires privés, etc. Même 
lorsque les institutions délivrent des certifications, l'examen des parcours d'élèves montrent que ceux-ci privilégient la formation à la certification, ne restant parfois qu'un an sur le programme qui les intéressait, ne passant pas toujours les examens de fins d'année malgré des résultats tout à fait corrects. Le deuxième exemple porte sur le fait de considérer implicitement les études d'histoire de la chimie qui ont été faites à partir de cas parisiens comme ayant une pertinence à l'échelle nationale. Il a été démontré que tel n'était pas le cas. Il y a certes une dissymétrie entre Paris et les provinces, notamment parce que c'est à Paris que sont situées les institutions marquant la reconnaissance et la réussite scientifique, mais cela n'implique pas que toutes les institutions parisiennes aient une envergure nationale et que les institutions des provinces aient une portée uniquement locale. Il faut reconsidérer Paris comme une ville de province, ce qui permettrait entre autres de faire un point sur l'ensemble des cours et des formations existants et qui pour l'instant sont occultés par les grandes institutions.

21 L'enjeu de ces projets de recherches est de changer le regard et le récit de l'histoire de la chimie en France, de ses acteurs, de ses savoirs, de son enseignement et de ses lieux, en prenant acte du renouvellement apportée par les études à l'échelle locale, en allant au-delà des institutions académiques et en articulant histoire des sciences et histoire des techniques.

Madame la présidente, mesdames et messieurs les membres du jury, je vous remercie pour votre attention.

\section{NOTES}

1. CRAWFORD Matthew B., Éloge du carburateur, Paris, La découverte/Poche, Kindle édition, 2016.

2. PROST Antoine, 12 leçons sur l'histoire, Paris, Éditions du Seuil, 1996

3. Ibidem, p.95.

4. CRAWFORD Matthew B., Éloge..., op.cit., 2016, loc 553.

5. Ibidem., loc. 3254 .

6. Ibid., loc. 3260 .

7. Ibid.

8. Ibid.

9. PROST Alain, 12 leçons..., op.cit., 1996, p.146.

10. Ibidem.

11. Ibid, p.147.

12. Ibid.

13. Voir en particulier, SCHILLING Pascal, VAN WICKEREN Alexander, « Towards a Material and Spatial History of Knowledge Production. An Introduction », Historical Social Research, vol.40, $\mathrm{n}^{\circ} 1$, 2015, p.203-218.

14. CRAWFORD Matthew B., Éloge..., op.cit., 2016, loc. 695.

15. Renaud d'Enfert a travaillé cette question pour le domaine des mathématiques: D'ENFERT Renaud, Pour une histoire " par en bas " de l'enseignement des sciences (XIX et $\mathrm{XX}^{e}$ siècle). Le cas des mathématiques, mémoire d'habilitation à diriger des recherches, université Paris Sud, 2012. C'est 
aussi un des objectifs du séminaire que nous avons organisé ensemble: l'offre locale d'enseignement scientifique et technique, et qui va donner lieu à un ouvrage collectif : D'ENFERT Renaud, FONTENEAU Virginie, L'offre locale d'enseignement scientifique et technique, 19e-20e siècle: approches disciplinaires, Parution prévue en 2019 aux Presses Universitaires de Nancy.

\section{RÉSUMÉS}

L'enjeu de cette recherche reposant sur une articulation entre histoire des sciences et histoire des techniques est d'examiner à nouveau frais le contraste entre deux territoires, d'une part, Paris, où les cours seraient nombreux, de haut niveau, et délivrés par des chimistes brillants et prestigieux, et d'autre part les provinces, où il y aurait un déficit d'intérêt pour la science en général, et donc pour son enseignement, ce qui provoquerait un départ pour la capitale de tout chimiste souhaitant faire carrière. Cette analyse repose sur une approche locale et la notion de système local, en ne sélectionnant pas a priori ce qui serait un enseignement de chimie/science et un enseignement de chimie/technique. Cette analyse met en évidence des acteurs absents de l'historiographie, qui existent et font carrière en dehors des institutions académiques, sont des producteurs de savoirs scientifiques et techniques, et pour beaucoup sont investis dans une activité d'entrepreneurs. Ce résultat conduit à revisiter l'émergence de la profession d'ingénieurchimiste au tournant du XXe siècle.

The challenge of this study, based on an articulation between the history of science and the history of technology, was to examine with fresh eye the contrast between two territories, Paris on the one hand, where courses would be numerous, of high level, and delivered by brilliant and prestigious chemists, and the rest of France on the other hand, where there would be a deficit of interest for science in general and for its teaching in particular, with the result that any chemist wishing to make a career, should leave for the capital. We have chosen another point of view to conduct our analysis, favoring the local approach with the notion of "local system", and refusing to make an "a priori" selection between what would be a chemistry teaching centered on science and a chemistry teaching focused on technology. We have thus been able to highlight actors totally absent from historiography, who have made a career outside academic institutions, who have been producers of scientific and technical knowledge, and who, for many of them, have invested themselves in an entrepreneurial activity. This leads to revisit the emergence of the profession of chemical engineer at the turn of the 20th century.

\section{INDEX}

Mots-clés : histoire des techniques, histoire des sciences, chimie, mécanique, enseignement

Keywords : history of technology, history of science, chemistry, mechanics, education

Thèmes : Positions de thèse/HDR 


\section{AUTEUR}

\section{VIRGINIE FONTENEAU}

Maîtresse de conférences en épistémologie, histoire des sciences et des techniques, Virginie Fonteneau dirige le Groupe d'Histoire et de Diffusion des sciences d'Orsay (GHDSO), au sein Laboratoire Études sur les Sciences et les Techniques (EST), EA 1610 à l'Université Paris-Sud/ Université Paris-Saclay. Ses travaux de recherche en cours portent sur deux axes, l'histoire de l'enseignement scientifique et technique et l'histoire de la chimie $\left(19^{\mathrm{e}}\right.$ et $20^{\mathrm{e}}$ siècle). Elle travaille en particulier sur les ingénieurs et la formation des ingénieurs en France ainsi que sur les acteurs, savoirs et lieux de formation à la chimie. 